Bobeth-Neumann, Wiebke

\title{
Angebote zur Qualifizierung für den Schulleitungsberuf. Deutungsmuster von Lehrkräften auf dem Weg ins Schulleitungsamt
}

Zeitschrift für interpretative Schul- und Unterrichtsforschung 4 (2015) 1, S. 92-105

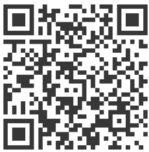

Quellenangabe/ Reference:

Bobeth-Neumann, Wiebke: Angebote zur Qualifizierung für den Schulleitungsberuf. Deutungsmuster von Lehrkräften auf dem Weg ins Schulleitungsamt - In: Zeitschrift für interpretative Schul- und Unterrichtsforschung 4 (2015) 1, S. 92-105 - URN: urn:nbn:de:0111-pedocs-153425 - DOI:

10.25656/01:15342

https://nbn-resolving.org/urn:nbn:de:0111-pedocs-153425

https://doi.org/10.25656/01:15342

in Kooperation mit / in cooperation with:

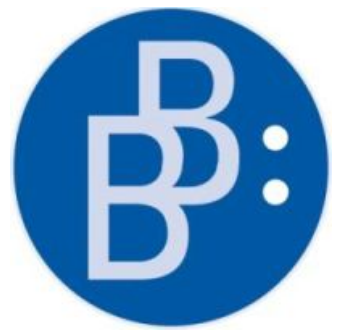

https://www.budrich.de

\section{Nutzungsbedingungen}

Gewährt wird ein nicht exklusives, nicht übertragbares, persönliches und beschränktes Recht auf Nutzung dieses Dokuments. Dieses Dokument ist ausschließlich für den persönlichen, nicht-kommerziellen Gebrauch bestimmt. Die Nutzung stellt keine Übertragung des Eigentumsrechts an diesem Dokument dar und gilt vorbehaltlich der folgenden Einschränkungen Auf sämtlichen Kopien dieses Dokuments müssen alle Urheberrechtshinweise und sonstigen Hinweise auf gesetzlichen Schutz beibehalten werden. Sie dürfen dieses Dokument nicht in irgendeiner Weise abändern, noch dürfen Sie dieses Dokument für öffentliche oder kommerzielle Zwecke vervielfältigen, öffentlich ausstellen, aufführen, vertreiben oder anderweitig nutzen.

Mit der Verwendung dieses Dokuments erkennen Sie die Nutzungsbedingungen an.

\section{Terms of use}

We grant a non-exclusive, non-transferable, individual and limited right to using this document.

This document is solely intended for your personal, non-commercial use. Use of this document does not include any transfer of property rights and it is conditional to the following limitations: All of the copies of this documents must retain all copyright information and other information regarding legal protection. You are not allowed to alter this document in any way, to copy it for public or commercial purposes, to exhibit the document in public, to perform, distribute or otherwise use the document in public.

By using this particular document, you accept the above-stated conditions of use.

\section{Kontakt / Contact:}

peDOCS

DIPF | Leibniz-Institut für Bildungsforschung und Bildungsinformation Informationszentrum (IZ) Bildung

E-Mail: pedocs@dipf.de

Internet: www.pedocs.de

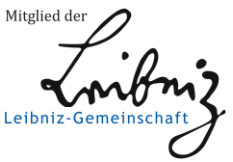




\section{Inhalt}

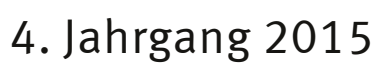

ZISU

\section{Zeitschrift für interpretative Schul- und Unterrichtsforschung}

\section{Editorial}

Ulrich Gebhard, Merle Hummrich, Kerstin Rabenstein, Sabine Reh

\section{Thementeil}

Georg Breidenstein

Thomas Grunau, Mirja Kekeritz

Matthias Martens, Barbara Asbrand, Christian Spieß

Julia Demmer

Jutta Wiesemann, Jochen Lange
Räume, Dinge und schulisches Wissen. Materials

„Verdammtes Ding, dich könn’ wir dann erforschen!“ Perspektiven auf kindliche Auseinandersetzungen mit den Dingen in institutionellen Kontexten

Lernen mit Dingen - Prozesse zirkulierender Referenz im Unterricht

Selbstpräsentationen von Zeitzeug_innen.

Fallrekonstruktion Schule

„Education in a Box“ Die Herstellung schulischer

80

Artefakte in der Lehr-Lernmittelindustrie

\section{Allgemeiner Teil}

Wiebke Bobeth-

Neumann

Anne Niessen
Angebote zur Qualifizierung für den

Schulleitungsberuf: Deutungsmuster von Lehrkräften auf dem Weg ins Schulleitungsamt

Musikpädagogische Perspektiven auf Heterogenität 106 und die Ambivalenz der Anerkennung. Ergebnisse einer qualitativen Interviewstudie mit Lehrenden des Programms ,Jedem Kind ein Instrument“ 
Sammy Wafi, Markus Wirtz

\section{Rezensionen}

Svenja Strauß

Till-Sebastian Idel
Visualisierungskompetenz in Deutsch und Mathematik aus Sicht von Expertinnen und Experten der Lehr-/Lernforschung und Fachdidaktik

Kade, Jochen/Nolda, Sigrid/Dinkelaker, Jörg/

Herrle, Matthias (Hrsg.) (2014): Videographische

Kursforschung. Empirie des Lehrens und Lernens

Erwachsener. Stuttgart: Kohlhammer Verlag

Zeitler, Sigrid/Heller, Nina/Asbrand, Barbara (2012): Bildungsstandards in der Schule. Eine rekonstruktive Studie zur Implementation der Bildungsstandards. Münster/New York/München/Berlin: Waxmann. ISBN 978-3-8309-2664-1. 


\title{
Allgemeiner Teil
}

\author{
Wiebke Bobeth-Neumann
}

\section{Angebote zur Qualifizierung für den Schulleitungsberuf Deutungsmuster von Lehrkräften auf dem Weg ins Schulleitungsamt}

\section{Zusammenfassung}

In vorliegendem Beitrag wird der Frage nachgegangen, welche Deutungsmuster Lehrkräfte, die den Aufstieg ins Schulleitungsamt planen, den bestehenden Qualifizierungsangeboten zugrunde legen und welche Vorstellungen von Schulleitungshandeln damit verknüpft sind. Der Beitrag fokussiert auf das Bundesland Schleswig-Holstein, in dem zwei fakultative Angebote zur Wahl stehen. Anhand von Interviewsequenzen sowie ethnographischen Ausschnitten und deren Analyse mittels dokumentarischer Methode kann gezeigt werden, dass die Lehrpersonen in ebendas Qualifizierungsangebot einmünden, welches mit ihrem Deutungsmuster bestmöglich harmoniert. Im Beitrag werden zwei Deutungsmuster einander gegenübergestellt. In einem bildet sich die Bezugnahme auf einen hohen Stellenwert von Wissenschaftlichkeit im Qualifizierungskurs ab sowie ein an hierarchischen Strukturen orientiertes Führungsverständnis. Dem zweiten Deutungsmuster liegt sowohl die Auffassung von Schulleitung als primus inter pares zugrunde als auch die Priorisierung einer Qualifizierung mit handlungspraktischem Trainingscharakter.

Schlagwörter: Qualifizierungsstruktur; Schulleitungshandeln; Deutungsmuster; Dokumentarische Methode

\section{Qualification opportunities for school leadership: patterns of interpretation of aspiring head teachers}

This article answers the question how teachers, who want to advance to the position of headmaster, value the current offers by institution to professionalise the inauguration. Furthermore their conceptions of headmaster agency can be shown. The results focuse on the federal state Schleswig-Holstein, where two optional offers stand for the choice. For the analysis interview sequences and ethnographic field notes were evaluated by the documentary method. It is evident, that the choices and the anticipations correspond with the patterns of interpretation (Oevermann: Deutungsmuster). The prospective headmasters choose the qualification structure, which seems to be in accordance with their patterns of interpretation. This article presents two patterns of interpretation. The first is oriented towards a significant value of scientific approach as well as towards a chain of authority. The second represents the concept of primus inter pares and the prioritization of being qualified by a training based on practical work.

Keywords: Qualification structure; school leadership; patterns of interpretation; documentary method

\section{Sich wandelnde Anforderungen an Schulleiter/innen}

Das Amt der Schulleitung beinhaltet verantwortungsvolle, jedoch nicht einheitlich definierte und sich stetig wandelnde, Aufgabenbereiche. Der Allgemeine Schulleitungsverband Deutschlands (ASD) konstatierte bereits 1994, „dass in keinem Bundesland eine dezidierte Zusammenstellung der Tätigkeiten von Schulleitung existiert“" (ASD 1999: 41). Ebenso wenig transparent sind die benötigten Kompetenzen für diese Tätigkeit. 
Die wissenschaftliche Auseinandersetzung mit den Anforderungen an Schulleitungshandeln und die dafür voraussetzungsvollen Fähigkeiten reicht in Deutschland bis in die Anfänge der 1980er Jahre zurück. Zu dieser Zeit wurde die Leitung einer Schule erstmals als eigenständiges Berufsbild mit spezifischen Anforderungen und nicht länger als ergänzende Verwaltungstätigkeit einer Person definiert, die ursprünglich für den Lehrberuf qualifiziert wurde (Wolfmeyer 1981). Empirische Forschungen untersuchten zunächst das Rollenverständnis von Schulleitungen sowie deren Akzentsetzungen und Einflussmöglichkeiten innerhalb des breiten Tätigkeitsfeldes (Baumert/Leschinsky 1986). Von besonderem Interesse war dabei stets die Gestaltung der pädagogischen Arbeit im schulischen Alltag.

Seit Beginn der 1990er Jahre erweiterte sich das Forschungsspektrum bedeutend und es rückte allmählich ein verändertes Bild von Schulleitung in den Mittelpunkt der Wahrnehmung. Im Zuge der durch die Reformbestrebungen gestiegenen Autonomie von Schulen verstärkten sich die Möglichkeiten der Einflussnahme von Schulleitungen. Zusehends wurde ihre Rolle nicht mehr nur unter dem Aspekt der Gestaltung und Leitung von Schule im Alltagsbetrieb, sondern zusätzlich im Hinblick auf die prospektive Weiterentwicklung von Schule definiert. Managementkonzepte aus der Wirtschaft werden seither auf schulische Entwicklungsprozesse angewendet und die Schulleitung wird zur Schlüsselfigur für die erfolgreiche Analyse, Planung, Realisierung und Evaluation von Veränderungsmaßnahmen im Rahmen gelingender Schul- und Unterrichtsentwicklung (Brohm 2004: 174f.). Organisations-, Unterrichts- und Personalentwicklung sind dem gewandelten Verständnis nach nun die wesentlichen Handlungsfelder von Schulleitung (Rahm 2005: 55).

Mit diesem erweiterten und gestiegenen Anspruchspostulat wurde in den 1990er Jahren auch die bis heute aktuelle Diskussion um die Professionalisierung von Schulleitungen entfacht (Huber/Wissinger 2002). Durch die für Schulleitungstätigkeit fehlende Berufsausbildung kann Professionalität bei aufstiegsinteressierten Lehrkräften hinsichtlich der avisierten Position nicht per se vorausgesetzt werden. Der passende Rahmen, sich Selbstverständnis, Blickwinkel und Praktiken dieser Profession anzueignen, fehlt. „Die Berufsausbildung erfordert nicht nur den Erwerb von Fachwissen, sondern auch und vor allem die Ausbildung eines Professionshabitus“ (Wernet 2003: 37). Zudem erschwert die verschiedenartige Funktionseingebundenheit von Schulleitungen infolge der weiterhin bestehenden Unterrichtsverpflichtung einen gelingenden Rollenwechsel von der Lehrperson zur Führungskraft ${ }^{1}$.

Es lassen sich jedoch zahlreiche Impulse erkennen, den Schulleitungsberuf zu professionalisieren und Strukturen zu schaffen, die künftige Schulleitungen institutionalisiert auf ihre künftige Tätigkeit vorbereiten. Die internationale Forschung zeigt, dass die Aspekte Inhaltsausrichtung, zeitliche Strukturierung und Umfang sowie die Frage der Verortung dieser Qualifizierungsangebote in großer Unterschiedlichkeit beantwortet und in die Praxis umgesetzt werden (für einen internationalen Überblick Huber 2003).

Aber auch in den 16 deutschen Bundesländern differieren diese Angebote hinsichtlich ihrer Freiwilligkeit oder Verpflichtung ebenso wie mit Blick auf Umfang

1 Insbesondere Schulleitungen an deutschen Grundschulen wird ein knapper zeitlicher Umfang für die Ausübung ihrer Leitungstätigkeit gewährt. Je nach Schulgröße stehen ihnen ca. 7-15 Verwaltungsstunden wöchentlich zur Verfügung. 
und Inhaltsstruktur. Die zahlreichen Modifikationen, die in jüngster Zeit in einzelnen Bundesländern erfolgten, verweisen darauf, dass es sich um einen noch unvollendeten Reformprozess handelt. Zudem kann konstatiert werden, dass das Absolvieren einer akademisierten Ausbildung für die Übernahme des Schulleitungsamtes nach wie vor zumeist auf der freiwilligen Bereitschaft der Aufstiegsaspirantinnen und -aspiranten basiert. Ebendiese Freiwilligkeit und Angebotsvielfalt ermöglicht potentiellen Schulleitungen derzeit unterschiedliche Zugriffsweisen. Der Blickwinkel darauf, Struktur und Handlung in ihrer Wechselwirkung zu analysieren, scheint demzufolge interessant.

\section{Relation impliziter Deutungsmuster von Lehrkräften und impliziter Konstruktionen von Weiterbildungsangeboten}

Der vorliegende Beitrag blickt aus der Perspektive potentieller Schulleitungen auf die bestehenden Möglichkeiten, die sich diesen Personen zum Erwerb der nötigen Schulleitungskompetenzen bieten. Dies geschieht vor der Folie der aktuellen Qualifizierungssituation des Bundeslandes Schleswig-Holstein. Dort gibt es derzeit zwei fakultative institutionalisierte Qualifizierungsmöglichkeiten: den berufsbegleitenden Fernstudiengang „Schulmanagement und Qualitätsentwicklung“ an der Universität zu Kiel sowie das „Training zur Vorbereitung auf Schulleitungsaufgaben (TVaS)“ des „Instituts für Qualitätsentwicklung an Schulen Schleswig-Holstein (IQSH)“.

Die nebeneinander existierenden Angebote stellen potentielle Schulleitungen vor eine Bewältigungsnotwendigkeit hinsichtlich einer Entscheidungsfindung. Ihrer Qualifizierungswahl - so die Vermutung - liegen Hintergrundüberzeugungen und Deutungsmuster zugrunde, die sowohl ihr Bild von Schulleitung wiederspiegeln als auch ihre implizite Orientierung, welcher Form der Qualifizierung diese bedürfen und welches Angebotskonzept diese Erwartung am ehesten erfüllt. Folglich dienen diese Deutungsmuster als Schlüssel zur Bearbeitung der Wahlsituation.

Darüber hinaus zeigt der Beitrag anhand einiger exemplarisch gewählter Beobachtungssequenzen implizite Konstruktionen der Weiterbildungsangebote selbst auf. Damit verbunden wird die Hypothese aufgeworfen, dass zwischen den Deutungsmustern der Akteure und den Bedeutungsstrukturen der Qualifizierungsprogramme möglicherweise ein Passungsverhältnis besteht.

Folglich lauten die zentralen Fragestellungen, denen dieser Beitrag nachgeht:

- Mit welchen Deutungsmustern belegen aufstiegsinteressierte Lehrkräfte zum einen den angestrebten Beruf, die Schulleitungsposition, und zum anderen die Qualifizierungsofferten?

- Inwieweit lässt sich aus den Rekonstruktionen der Qualifizierungsmaßnahmen auf deren implizite Doxa schließen? 


\section{Strukturierungstheorie nach Giddens und Oevermanns Deutungsmusterkonzept}

Für die Analyse der Deutungsmuster und die Rekonstruktion der Struktur zweier Qualifizierungsangebote wird auf Giddens Strukturierungstheorie sowie auf das Gegenstandskonzept der Deutungsmuster nach Oevermann zurückgegriffen. Nachfolgend werden die wesentlichen Potenziale beider Theorien kurz skizziert.

Giddens Begriff der Strukturierung umfasst die Termini Handlung und Struktur zugleich. Sein „structure-agency“-Ansatz begreift existente Strukturen nicht als dualistischen Gegenspieler des Handelns. „Die sozialen Systeme, in denen Struktur rekursiv einbegriffen ist, umfassen demgegenüber die situierten Aktivitäten handelnder Menschen, die über Raum und Zeit reproduziert werden“ (Giddens 1997: 77). Demzufolge vollzieht sich eine rekursive Reproduktion institutioneller Ordnungen in Handlung und Interaktion. Die Struktur ist Voraussetzung für und Ergebnis von Handlung zugleich. Bezogen auf die vorliegende Untersuchung bedeutet dies, dass sich aufstiegsbereite Lehrkräfte mutmaßlich nicht mehreren gleichwertigen Qualifizierungsalternativen gegenübergestellt sehen, eine erwählen und resultierend aus der Qualifizierungsform ihr Führungsverständnis etablieren. Vielmehr ist zu vermuten, dass sich in ihrem praktischen Bewusstsein bereits Vorstellungen eines Schulleitungs-Selbstbildes und einer Führungsideologie eingelagert haben. Die erwählte Qualifizierungsform wirkt als strukturierendes Element dieser Prädisposition, als passendes Handlungsformat. Folglich kann davon ausgegangen werden, dass die (angenommene) Beschaffenheit der gewählten Qualifizierungsalternative von den Agierenden als geeignete Anschlussmöglichkeit an ihre bereits bestehende Disposition bewertet und ihr daher maximale „Fazilität“ (Neuberger 1995: 321) unterstellt wird.

Ebendiese handlungsleitenden, impliziten Wissensbestände sollen in diesem Beitrag mit dem Oevermannschen Deutungsmusterkonzept als heuristischer Kategorie bearbeitet werden. Diesem entsprechend wird davon ausgegangen, dass Akteure entlang einer inneren Logik, einer ,wie selbstverständlich für gültig gehaltene(n) Orientierung“ (Oevermann 2001a: 43) handeln, die über das sprachliche Moment der Interviewbefragung offengelegt werden kann und rekonstruierbar wird. Deutungsmuster sind nach Oevermann in sich konsistent, insofern als sie „Entscheidungskriterien für die Akzeptabilität oder Angemessenheit von Interpretationen auch bisher unbekannter Ereignisse abgeben" (Oevermann 2001b: 10).

Die Grundannahme des Deutungsmusteransatzes, dass es sich bei dem aus Alltagserfahrungen generierten Wissensstand nicht um voneinander separierte Einzelerfahrungen handelt, sondern dieser einer „Kontingenzregel“ (Oevermann 2001b: 9) folgt und einer gewissen Beständigkeit unterliegt, lässt eine architektonische Analogsetzung dieses Ansatzes zu jenem der Orientierungsrahmen oder auch zum Habitusansatz von Bourdieu zu. Als weitere Parallele ist auch diesen beiden inhärent, dass dieses verinnerlichte Verstehens- und Bewertungsmuster nicht nur aus Vergangenem resultiert, sondern auch Künftiges beeinflusst. Sie sind demnach Wahrnehmungs- und Interpretationsmuster zugleich und gelten - auch das ist den obigen Ansätzen gemein - als bedingt reflexiv verfügbar.

Das Deutungsmusterkonzept nach Oevermann enthält verschiedene Anschlussmöglichkeiten methodologischer Art. Neben der objektiven Hermeneutik und der Deu- 
tungsmusteranalyse ebenso „fruchtbar erscheint die Methode der dokumentarischen Interpretation nach Ralf Bohnsack“ (Ullrich 1999: 432). Durch die signifikante Nähe des Deutungsmusterkonzepts zum Konstrukt der Orientierungsmuster und insbesondere durch die profunden Auswertungsschritte zur systematischen Kontrastierung unterschiedlicher Sinnstrukturen wurde in der Studie, auf die dieser Beitrag rekurriert, die dokumentarische Methode erwählt.

\section{Methodische Vorgehensweise}

Das herangezogene empirische Datenmaterial entstammt einer qualitativen Untersuchung aus den Jahren 2010 bis 2012 im Rahmen derer die Orientierungsrahmen aufstiegsbereiter Grundschullehrkräfte mittels Leitfadeninterviews eruiert wurden. Trianguliert wurden diese Daten mit ethnographischen Beobachtungen der beiden obig bereits erwähnten schleswig-holsteinischen Qualifizierungsangebote (V 2013).

Die Interpretation der bestehenden Struktur und das aus den Deutungsmustern der aufsteigenden Lehrkräfte resultierende Handeln kann als konjunktiver Erfahrungsraum verstanden und durch ein rekonstruktives Verfahren wie die dokumentarische Methode erschlossen werden. Das Interpretationsverfahren der dokumentarischen Methode wird in diesem Kontext dem der objektiven Hermeneutik bewusst vorgezogen, wenngleich sich beide ähneln. Ihnen gemein ist die Annahme, dass narrativem empirischem Forschungsmaterial stets über das explizit vermittelte hinaus ein impliziter Sinngehalt innewohnt. Die Rolle des Interpreten differiert jedoch zwischen beiden Auswertungsverfahren ebenso wie die des Erzählers. Während ein wesentlicher Analyseschritt der Sinnrekonstruktion im Rahmen der objektiven Hermeneutik darin besteht, ungenannte Optionen, Handlungsmöglichkeiten etc. experimentell an das Material heranzutragen, arbeitet die dokumentarische Methode weniger mit dem Erkenntnishorizont des Forschers, sondern geht zwei andere Wege: Zum einen wird durch den hohen Stellenwert, der Fokussierungsmetaphern (dramaturgische Verdichtungen oder Schlüsselstellen im empirischen Material) zukommt, eine größtmögliche Nähe zu den erzählerischen Bedeutungshöhepunkten möglich. Zum anderen drängt die Sequenzanalyse im Rahmen einer fallübergreifenden Analyse den Interpretationshorizont des Forschenden zurück. Der Unterschied beider Interpretationsverfahren hinsichtlich der Rolle des Erzählers besteht in der Latenz, die beide Verfahren dessen Wissen zugestehen. Entsprechend der objektiven Hermeneutik steuern latente Sinnstrukturen Handlungen hinter dem Rücken der Akteure, während gemäß der dokumentarischen Methode latente Sinngehalte durchaus ins Bewusstsein des Handelns gelangen können.

Das Ziel der vollzogenen Analyse besteht nicht in der Offenlegung einer normativen Richtigkeit der Beurteilung bestehender Qualifizierungsangebote. Im Fokus stehen stattdessen die Wirklichkeitskonstruktionen, die potentielle Schulleitungen über ihre Qualifizierungswahl und ihre künftige Position entfalten. Dieses implizite Wissen gilt es mit den folgenden aufeinander aufbauenden Schritten rekonstruktiv zu interpretieren und eine theoriebasierte Explikation der Orientierungsrahmen zu leisten.

Der erste Schritt, die formulierende Interpretation, besteht in der inhaltlichen Beschreibung einzelner Interviewsequenzen, die eine Strukturierung und thematische 
Gliederung des Materials ermöglicht. Dieser Schritt verbleibt auf inhaltlicher Ebene so nah am Zitat, dass auch signifikante Begriffe beibehalten werden (Bohnsack/NentwigGesemann/Nohl 2001). Beispielhaft wird der folgende Transkriptausschnitt wie unten stehend deskriptiv zusammengefasst:

Ich kann mich tausendmal darüber aufregen, was wir hier für Pfeifen rumlaufen haben, aber wenn man immer nur von unten schießt, ändert sich nichts. Also muss ich mich dahin begeben, wo man im Zweifelsfall so was auch bekämpfen kann. (Herr L., Z. 97-100)

Formulierende Interpretation: Herr L. erwartet von seinem positionellen Aufstieg eine Ausweitung seines Handlungsspielraumes, um gegen Personen, die er abwertet („,Pfeifen“), vorgehen zu können (,,bekämpfen“).

Während die formulierende Interpretation paraphrasierend zusammenfasste, fragt die reflektierende Interpretation, die den zweiten Schritt der dokumentarischen Methode darstellt, statt nach der Was-Ebene, nach der Art und Weise des Gesagten, der WieEbene der Erzählung oder Beschreibung (Nohl 2008: 48f.). In diesem Analyseschritt kann der Orientierungsrahmen eruiert werden. Der Rahmen, innerhalb dessen eine befragte Person ihre Argumentation aufbaut, bildet ein Sinnmuster, das sich in verschiedenen Interviewphasen unabhängig von Fragestellung und Thema reproduziert. Nicht das kommunikativ vermittelbare Wissen steht - wie im ersten Auswertungsschritt - im Mittelpunkt, sondern diejenigen als konjunktive Wissensbestände bezeichneten impliziten Orientierungen, die handlungsleitend wirken.

Bezugnehmend auf das obige Beispiel könnte ein reflektierender Interpretationsansatz lauten:

Im Modus der Argumentation wirft Herr L. an dieser Stelle zum ersten Mal seine Orientierung einer hohen Einflussnahme durch eine Führungsposition auf. Der Exemplifizierung, dass sich diese Einflussnahme auf eine von ihm abqualifizierte Personengruppe beziehen soll, wird an dieser Stelle noch keine Erläuterung hinzugefügt, worauf sich die Unzulänglichkeit dieser Personen bezieht und welche Intervention er avisiert.

Von einem reliabel identifizierten Orientierungsrahmen (bzw. Deutungsmuster) lässt sich folglich erst sprechen, wenn an thematisch unterschiedlichen Stellen des Interviews eine homologe Struktur herausgearbeitet werden konnte. Besonders deutlich lassen sich differente Orientierungsrahmen von Interviewproband/innen durch den Kontrast zu Interviewsequenzen anderer befragter Personen erschließen. Das zentrale Merkmal der dokumentarischen Methode stellt dementsprechend die komparative Analyse dar. Die komparative Analyse gilt als Teil der reflektierenden Interpretation, da sie in erkenntnisgenerierender und erkenntniskontrollierender Form den Prozess dieser Auswertungsphase beeinflussen soll. Die fallkontrastive Interpretationsweise kann letztlich durch die Explikation von Homologien und Kontrasten zu einer Typologie sozialer Deutungsmuster bzw. Orientierungsrahmen führen. Im Folgenden wird nicht auf die im Rahmen der Studie generierte Typologie rekurriert, die sich aus zahlreichen weiteren Parametern neben dem der Angebotsentscheidung speist (V 2013). Stattdessen werden im Fokus der Qualifizierungswahl zwei differente Deutungsmuster einander gegenübergestellt.

Ähnlich verhält es sich mit der Herangehensweise an das ethnographische Material. Die Beobachtungsbögen, die während der teilnehmenden Beobachtung der bei- 
den Qualifizierungsangebote in Schleswig-Holstein verfasst wurden, entsprechen im Verständnis der dokumentarischen Methode bereits der formulierenden Interpretation. Sie wurden sequenziell und im Rahmen eines intersubjektiven Austausches in einem Forschungscolloquium einer reflektierenden Interpretation unterzogen. Anschließend wurden thematisch vergleichbare Sequenzen nach homologen Mustern durchsucht, die eine institutionelle Charakteristik des jeweiligen Angebotes abbilden und fallübergreifend mittels der komparativen Analyse miteinanderverglichen (Bohnsack/NentwigGesemann/Nohl 2001: 251).

Bei der Untersuchung der Fortbildungsangebote auf die ihnen inhärente Spezifik handelt es sich um ein exploratives Vorgehen, zu dem erste Ergebnisse hier nur skizzenhaft angedeutet werden können. Der Analyse liegt die Annahme zugrunde, dass die jeweiligen Angebotsformen einen konjunktiven Erfahrungsraum bilden, innerhalb dessen sich ein identisches Deutungsmuster bezogen auf das Führungsverständnis und das berufliche Handeln von Schulleitungen vermittelt.

\section{Deutungsmuster I: Stellenwert von Wissenschaftlichkeit und Hierarchie}

Am Beispiel des 32jährigen Herrn L., der sich für das Schulleitungsamt qualifiziert, soll ein Deutungsmuster rekonstruiert werden, welches der Präferenz der Qualifizierung qua Masterstudiengang „Schulmanagement und Qualitätsentwicklung“ an der Christian-Albrechts-Universität zu Kiel zugrunde liegen kann. Herr L. befindet sich zum Zeitpunkt des Interviews im ersten Semester des viersemestrigen Studienganges. In der folgenden Sequenz begründet er seine Qualifizierungswahl:

\footnotetext{
„Das war eigentlich nur etwas, wo ich dachte, wenn ich mich dann irgendwann nach X zurückbewerbe und da gibt es Konkurrenten, dass man dann sagen kann: Zack, ich hab hier aber noch ein Pfund in die Waagschale zu werfen. Vielleicht sticht man dann andere aus, wenn man besser qualifiziert für diesen Beruf ist. (...) Das ist eine breitere Qualifizierung, so hab ich das gesehen. Und ein höherwertiger Abschluss mit mehr wissenschaftlichem Tiefgang. Und ich find das auch ganz interessant, weil es mal nicht IQSH ist. Also, man hat es ja schon so verkauft, dass es nicht IQSH ist. Wer hier in Schleswig-Holstein lange gelitten hat, der weiß, dass nicht alles vom IQSH toll ist. Es ist immer dieses Lehrer bilden Lehrer fort. Wie Herr Dr. X immer sagt, dieses primus inter pares. Wir sind alle auf einer Stufe und dann kommt einer, der sich irgendwie fortgebildet oder hochbeworben hat. Das hab ich also in meinem Referendariat grauenvollst erlebt, dass wir Leute hatten, die versucht haben uns was beizubringen und so schulfremd waren. Insofern war das für mich ein höherer Anspruch, zu sagen, da ist die Uni, die dahinter steht.“ (Z. 116-136).
}

Herr L. betont die Bedeutung, die seiner Interpretation zufolge einer profunden Qualifizierung für eine Bewerbungssituation zukommt. Er vertritt die Überzeugung, dass er seine Erfolgsaussichten auf die Übernahme einer Führungsposition durch das Masterstudium und insbesondere durch dessen ,Wissenschaftlichkeit“ beträchtlich erhöhen kann. Seiner Auffassung nach braucht es ,wissenschaftlichen Tiefgang“, um qualifiziert im Schulleitungsamt handlungsfähig zu sein. Den passenden Rahmen zur Vermittlung dieser Wissenschaftlichkeit stellt der universitäre Kontext dar. Alle befragten potentiellen Schulleitungen, die in diesem Deutungsmuster verortet werden konnten, fühlen sich 
vom universitären Feld besonders angezogen, da dieses für sie mit der Erwartung einer qualitativ hochwertigeren Ausbildung assoziiert ist.

Der Interviewausschnitt von Herrn L. dokumentiert darüber hinaus, dass die dem Masterstudiengang zugeschriebene Wissenschaftlichkeit vor dem negativen Gegenhorizont der Wahlalternative konkretisiert wird. Herr L. stützt sich in seiner Argumentation vorwiegend auf den „Primus-inter-pares-Mythos“ (Schratz 1998: 161). Eine Struktur, die es vermag, Personen mittels wissenschaftlich fundierter Kenntnis für ein Amt zu qualifizieren, bedarf nicht nur des passenden Ortes, Inhaltes und Zeitumfanges, sondern braucht ebenso äquivalente Lehrende. Für mögliche Bewerber/innen um einen Schulleitungsposten, die das Deutungsmuster Wissenschaftlichkeit aufweisen, können diese Lehrenden keine weitergebildeten Lehrkräfte sein. Stattdessen ist die Legitimation von Wissenschaftlichkeit gebunden an Hochschullehrende, also an Personen, die in der Wahrnehmung der Lehrkräfte hierarchisch höher zu klassifizieren sind. Die Erwünschtheit von Hierarchie lässt bereits erste Rückschlüsse auf die Wechselwirkung zwischen der Wahl der Qualifizierungsstruktur und der künftigen Handlungspraxis dieser potentiellen Schulleitungen zu: Das Deutungsmuster Wissenschaftlichkeit ist mit einem ausgeprägten Führungs- und Hierarchieverständnis verzahnt. Die Zielorientierung, im Sinne eines hierarchischen Führungsverständnisses für die Schulleitungstätigkeit qualifiziert zu werden, ist jedoch nicht im Bewusstsein der potentiellen Schulleitungen vorhanden. „Ein Handelnder muss sich nicht des Ziels bewußt sein, das er zu erreichen sucht. Das meiste des Handlungsstroms, der das Alltagshandeln konstituiert, ist in diesem Sinne vor-reflektiert" (Giddens 1984a: 91). Während sich der Wunsch nach einer an Wissenschaftlichkeit orientierten Struktur als durchaus der Sprache zugänglich erweist und demzufolge als ,diskursives Bewusstsein“ (Giddens 1997: 429) verstanden werden kann, handelt es sich bei der Korrelation zwischen dem eigenen Führungsverständnis und der vollzogenen Qualifizierungswahl um praktisches Wissen (praktisches Bewusstsein). Dieses wird nicht über die Sprache zum Ausdruck gebracht, sondern bildet sich im Handlungsvollzug ab. Hier zeigt sich auch das Deutungsmusterkonzept von Oevermann anschlussfähig, welches von implizitem Wissen spricht, wenn Wissen ,als nicht abfragbares, aber, wie auch immer bedingt, unbewußt operierend in die Praxis strukturierend eingeht" (Oevermann 2001a: 56).

Dazu erfolgt exemplarisch eine Analyse des Führungsverständnisses von Herrn L.

„Ich glaube, Führung hat nicht viel mit Lehrerfahrung zu tun. (...) Man hat irgendwie weniger Kontakt und dieses informelle Türangelgespräch entfällt irgendwie. (...) Also, es gibt hier eine Kollegin, wenn die Vertretungsbereitschaft hat in der ersten Stunde, dann wird die immer angerufen, wenn sie kommen muss. Vertretungsbereitschaft ist Vertretungsbereitschaft. Darüber würde ich nicht diskutieren. Wenn sie dann hier ist und muss nicht unterrichten, dann kann sie ihren Unterricht mal vorbereiten. Ansonsten vertritt sie.“ (Z. 336-363)

In obiger Aussage lässt sich eine Grundhaltung erkennen, die darauf hinweist, dass Herr L. strukturelle Zwänge, die eine Schulleitung umzusetzen hat, nicht als determinierend bewertet. Vielmehr dienen ihm formelle Befugnisse als Bezeugung seiner Führungsberechtigung. Sein Führungsverständnis scheint geprägt von formaler Autorität und geringer Interaktionsdichte zwischen Führungsperson und Lehrkräften. Eine Reduktion des kollegialen Kontaktes und eine Weisung gegen Widerstand kann von ihm ohne Defizitempfinden registriert werden. „Struktur darf nicht mit Zwang gleichgesetzt werden: 
sie schränkt Handeln nicht nur ein, sondern ermöglicht es auch“ (Giddens 1988: 77f.). Wenn davon auszugehen ist, dass Strukturen ,interaktive Beziehungen über Raum und Zeit stabilisieren“ (Walgenbach 2006: 410), so können sie ebenso dem Zweck dienen, Hierarchien zu stabilisieren. Die obige Interviewsequenz gibt einen Anhaltspunkt dafür, dass die Struktur des Masterstudienganges, die eine spürbare Rangordnung aufweist, von den studierenden Lehrkräften vermutlich im eigenen Schulleitungshandeln reproduziert wird. Hier realisieren sich Strukturmomente in sozialer Praxis: Kommunikation, Ausübung von Macht und die Bewertung von Verhaltensweisen auf normativer Basis - die Dimensionen der Dualität von Struktur nach Giddens (1997: 81) - stehen in untrennbarer Verbindung miteinander und bilden die soziale Praxis von Personen, die diese Qualifizierungsmaßnahme favorisieren.

Um die Ergebnisdarstellung noch zu ergänzen und zu sublimieren, soll abschließend ein kurzer Exkurs in die Daten der ethnographischen Erhebung im Rahmen des Masterstudienganges erfolgen (ausführlich V 2013). Sowohl die analysierten Dokumente (bspw. der Studiengangsflyer) als auch die Beobachtungsprotokolle lassen erste Rückschlüsse darauf zu, dass dem Studiengang ein generatives Prinzip zugrunde liegt, welches mit dem Deutungsmuster „Stellenwert von Wissenschaftlichkeit und Hierarchie" harmoniert und die Hypothese eines möglichen Passungsverhältnisses von Angebots-Bedeutungsstruktur und Proband/innen-Deutungsmustern stützt.

Der Studiengang versteht sich als Vermittler „wissenschaftlicher Grundlagen für pädagogische Führungstätigkeit, relevanter Methoden und Ergebnisse der empirischen Schul- und Unterrichtsforschung (sowie) professioneller Kompetenzen in den Bereichen Qualitätsmanagement, Personalführung und Kommunikation" (Flyer zum Studiengang 2012). Die Betonung der Wissenschaftlichkeit wird zudem dadurch unterstrichen, dass die Präsenzseminare durch Vorträge führender Wissenschaftler in Form einer Vorlesung im Hörsaal gerahmt werden. Das Vorherrschen eines ausgeprägten Hierarchieverständnisses dokumentiert sich in der stetigen Anspracheform der Studierenden als „künftige Führungskräfte“. Exemplarisch soll hier auf ein Beispiel zurückgegriffen werden, welches die charakteristische Praxis abbildet (ausführlicher dazu: V 2013: 145ff.):

Eine Dozentin und ein Dozent, formell gekleidet, betreten den Raum und begrüßen die Studierenden. Diese werden während der gesamten Einführungsveranstaltung gesiezt und wiederholt als „künftige Führungskräfte“ tituliert. Beispielsweise beginnt der Dozent seine Sätze häufig mit der Anrede „Sie als künftige Führungskräfte“ (PrMa, Z. 437-445).

In der sozialen Praxis der Dozierenden verwirklicht sich die Struktur des Qualifizierungsangebotes, welche nach Giddens definiert ist als ,rules and resources, recursively implicated in the reproduction of social systems" (Giddens 1984b: 377).

\section{Deutungsmuster II: experimenteller Praxisbezug und Kollegialität}

Die zweite Qualifizierungsmöglichkeit für angehende Schulleitungen in SchleswigHolstein ist das seit 2009 bestehende ,Training zur Vorbereitung auf Schulleitungsaufgaben (TVaS)“ des „Instituts für Qualitätsentwicklung an Schulen Schleswig-Holstein 
(IQSH)“. Dieses wird in modularisierter Form an unterschiedlichen Standorten angeboten, kann in zeitlicher Struktur und Reihenfolge von den Teilnehmenden individuell kombiniert werden und hat einen Gesamtumfang von 136 Stunden. Die Modulleiter/ innen sind überwiegend Lehrkräfte, die sich durch eine Ausbildung zum Coach, Supervisor oder Mediator für diese Tätigkeit qualifiziert haben.

Am Beispiel der 56jährigen Frau K. soll das Deutungsmuster experimenteller Praxisbezug und Kollegialität erläutert werden. Frau K. hatte zum Zeitpunkt des Interviews das ,TVaS“ absolviert und war gerade in die Schulleitungsposition aufgestiegen. Sie schildert ihren Eindruck vom ,TVaS“:

„Das hab ich ja mit 'ner Freundin zusammen gemacht, die jetzt auch weg ist und auch Schulleitung geworden ist. (...) Das war auch wirklich nett, war auch wirklich gut. Hat auch viel gebracht. Ich hab das letzten Sommer, nee, ich hab, glaub ich, schon vor dem Sommer angefangen. Ich hatte schon mal mit diesem ,Unterricht beurteilen“ angefangen. Dann hatte ich danach noch ,Gesprächsführung'. Ich hab gedacht: Das ist sowieso gut. Also ,Unterricht beurteilen“ wenn ich 'ne Referendarin nehmen will, muss ich das sowieso noch mal machen, um das zu können. Und ,Gesprächsführung“ ist auch immer gut. Da macht man auch Rollenspiele und kann sich mal ausprobieren und so. Mal gucken: Ja, wie würde ich da denn jetzt reagieren?“ (Z. 140-153).

Frau K. führt die inhaltliche Schwerpunktsetzung mehrerer Module auf und interpretiert die Struktur des „TVaS“ vornehmlich auf der Ebene des experimentellen Übens mit hohem Praxisbezug. Als unmittelbaren Einsatzbereich der erlernten Fähigkeiten benennt sie keine spezifisch führungsrelevanten Handlungsfelder, sondern alltägliche Aufgabenbereiche von Lehrkräften: die Unterrichtsbeurteilung und die pädagogische Gesprächsführung. Im Gegensatz zu Herrn L. zielt ihre Argumentation für oder gegen ein Qualifizierungsangebot nicht auf den konkreten Aspekt der Bewerbungschancen oder spezielle Qualitätskriterien des Angebotes ab. Zwar avisiert Frau K. einen Kompetenzzuwachs, sie grenzt diesen jedoch nicht von ihrer vorigen Tätigkeit als Lehrerin ab. Ihr Deutungsmuster, hier bezeichnet als experimenteller Praxisbezug, begreift den Schulleitungsberuf nicht als eigenständigen Beruf, für den es eines speziellen Expertenwissens bedarf. Sie generiert das benötigte Handlungswissen auf Grundlage ihrer Erfahrungen. Die Struktur des „TVaS“ dient ihr dabei - im Sinne der Strukturierungstheorie von Giddens - zur Reproduktion und Weiterführung ihrer Routinen. Die vorgefundene Struktur des „TVaS“ eignet sich einerseits dazu, das eigene Deutungsmuster zu rechtfertigen: Es ist eine Struktur existent, die ein praxisorientiertes Angebot bietet; folglich erscheint diese Schwerpunktsetzung als adäquate Vorbereitung auf künftiges Schulleitungshandeln. Andererseits werden so die Strukturmomente des Angebotes reproduziert: ,Actors draw upon the modalities of structuration in the reproduction of systems of interaction, by the same token reconstituting their structural properties“"(Giddens 1984b: 28).

Potentielle Schulleitungen, deren Zugang zur Wahl der Qualifizierungsform dem Deutungsmuster des experimentellen Übens entspricht, nutzen die Vorab-Qualifizierung für eine weitläufige Kompetenzerweiterung. Um diese zu erreichen, haben sie auf didaktischer Ebene eine konkrete Erwartung an das Angebot: Sie wollen innerhalb des Lern-Settings handlungsorientiert und praxisbezogen arbeiten. Bezüglich der fachlichen Kompetenz der Lehrenden findet hingegen keine explizite Auseinandersetzung statt: Sie wird weder als besonders relevant hervorgehoben noch kritisch hinterfragt. Dieses 
Deutungsmuster harmoniert mit einem Führungsverständnis, welches Kollegialität priorisiert. Exemplarisch kann dies an der folgenden Sequenz gezeigt werden, in der Frau K. ihr Erleben als neu ernannte Schulleiterin beschreibt:

„Ich sitz da im Lehrerzimmer zwar meistens, wenn Pause ist, aber ich weiß, ich gehör da ja nicht so richtig zu. Ich bin hier etwas einsam. Das hab ich auch gestern gerade mit der Freundin, die da in X ist,- also dass wir im Grunde so zwischen allen Stühlen stehen als Schulleitung. Das war mir vorher nicht so klar, obwohl man sich das eigentlich hätte denken können. Also, so langsam wird es besser. Aber kann sein, wenn von Kiel was kommt und ich was durchdrücken muss gegen die Kollegen, dann bin ich wieder draußen, also aus dem Klüngel. Das ist nicht gut. Das fällt mir auch schwer. Das ist nicht so schön. (...) Wir sind acht, nee neun Kolleginnen. Ich glaub, bei zweien von denen war ich noch nie zu Hause. Bei allen anderen war ich schon zu Hause oder sie bei mir.“ (Z. 220-240)

Die neue Rolle der Schulleitung wird in Frau K.s Darlegung vornehmlich auf der Beziehungsebene exemplifiziert. Die von ihr angeführten Beispiele beziehen sich auf informelle Kontaktaufnahmeversuche zu ihren Kolleginnen, die sie in Schulpausen oder außerhalb der Schule wahrnimmt. Dabei deuten ihre Formulierungen eher darauf hin, dass ihr Selbstverständnis nach wie vor primär dem einer Lehrkraft als dem einer Vorgesetzten entspricht. Die Umbruchssituation stellt für sie bedingt durch ihr Deutungsmuster eine krisenhafte Herausforderung dar. Statt der empfundenen Einsamkeit möchte sie im „Klüngel“ drin sein und gerät demzufolge bei oktroyierten Anordnungen in einen Rollenkonflikt, da sie ihre Weisungsberechtigung als den intensiven kollegialen Kontakt konterkarierend erlebt. Die Äußerung ,, das war mir vorher nicht so klar “ lässt sich als nicht-antizipierte Konsequenz ,,auf der Ebene der institutionellen Rahmenbedingung (interpretieren), (die) die Handlungssubjekte vor deutungsbedürftige Probleme (stellt)“ (Oevermann 2001b: 22). Ihr Führungsverständnis ist geprägt von dem Wunsch nach einer konsensfähigen Gestaltung der Arbeitsbeziehung und einer Ergänzung dieser durch eine ins Private hineinreichende intensive Sozialbeziehung. Die inhärente Asymmetrie im Verhältnis zwischen Lehrkräften und Schulleitungen können Personen dieses Deutungsmusters nur schwer akzeptieren. Die vereinzelnde Struktur, innerhalb derer Schulleitungen agieren, wird als belastend bewertet.

Die Kombination des Wunsches, sich an künftige Schulleitungsaufgaben experimentell und praxisorientiert heranzutasten, mit dem Ansinnen, einen Führungsstil mit persönlichen, dezentralen Kommunikationsstrukturen zu pflegen, kann unter dem Fokus auf Deutungsmuster als eine generative Strukturlogik aufgefasst werden. Diese tiefenstrukturelle innere Logik sucht eine Struktur, in der sie sich ,,heimisch fühlt“ und entfalten kann. In den Beobachtungsprotokollen finden sich Anzeichen dafür, dass das Deutungsmuster des experimentellen Praxisbezugs und der Kollegialität sich sowohl in das Selbstverständnis des „TVaS“ einpasst als auch von den daran Teilnehmenden kollektiv geteilt wird. Bereits die Bezeichnung „Training“ impliziert ein längerfristiges und möglicherweise praxisorientiertes Üben. So äußert eine Teilnehmerin im Rahmen eines Moduls:

„Ich will üben, Schulleiterin zu werden, irgendwann mal. Ich bin jetzt schon im dritten Jahr beim ,TVaS“.“ (PrTVaS, Z. 264-266) 
Die folgende Beobachtungssequenz konkretisiert abschließend das von Institution und Personen geteilte Deutungsmuster und die daraus resultierende Erzeugung einer sozialen Gemeinschaft:

Nach weiteren Rollenspielen und der Mittagspause inszenieren zwei Teilnehmende ein Rollenspiel(...). Im Anschluss wird dieses beklatscht und kommentiert. Die Teilnehmenden sprechen durcheinander und verzichten auf Handzeichen, um ihren Redebeitrag anzukündigen. (...) Gelegentlich fallen die Teilnehmenden auch dem Dozenten ins Wort oder widersprechen ihm. Es wird durchgängig die „Du-Form“ verwendet. (PrTVaS, Z. 220-237)

Wenngleich die Reproduktion einer Struktur nicht in einer einzigen isolierten Handlung zu erkennen ist, lässt die obige Beobachtung dennoch Rückschlüsse auf die im Handeln angewendete implizite Konstruktion des „TVaS“ zu. Diese scheint an flachen Hierarchien und positionsübergreifender Kollegialität orientiert zu sein und in ihrer Bedeutungsstruktur an das Deutungsmuster ,experimenteller Praxisbezug und Kollegialität“ anzuschließen.

\section{Diskussion der Ergebnisse}

In vorliegendem Beitrag wurden Deutungsmuster herausgearbeitet, um einen Erklärungsansatz für das Wahlverhalten potentieller Schulleitungen in Schleswig-Holstein anzubieten. Um soziale Deutungsmuster zu analysieren, ist nach Oevermann ,die Identifikation der krisenhaften Reibungsflächen zwischen der ,inneren Logik` der Deutungsmuster und den von den objektiven Strukturbedingungen gestellten Handlungsproblemen wesentlich“ (Oevermann 2001b: 23). Die Ergebnisse skizzieren die divergenten Erwartungen, die potentielle Schulleitungen an die beiden Qualifizierungsmöglichkeiten stellen und die Foki, unter denen sie die Angebote bewerten. Die Rekonstruktion der Deutungsmuster konnte zeigen, dass die befragten Personen in eine Angebotsstruktur einmünden, die ihrem praktischen Bewusstsein nach als übereinstimmend zur Tiefenstruktur ihres Handelns wahrgenommen wird. In dieser Hinsicht können die vorliegenden Befunde als vorläufige Bestätigung der Hypothese gewertet werden, dass Schulleitungs-Deutungsmuster und Bedeutungsstrukturen der Qualifizierungsprogramme von Seiten der potentiellen Schulleitungen aufeinander abgestimmt werden. Personen, die über ein eher hierarchisches und autoritäres Führungsverständnis verfügen, bevorzugen den Befunden zufolge ein Qualifizierungsangebot, in welchem sie bereits als künftige Führungskräfte wahrgenommen werden, dem sie hohe Wissenschaftlichkeit sowie Vorteile in der Bewerbungssituation zuschreiben. Aufsteigende Lehrkräfte, die sich sehr kollegial verorten, scheinen Schulleitungshandeln als praktisch erprobbar zu bewerten und bevorzugen ein niedrigschwelliges Übungsszenario.

Die Ergebnisse verweisen darauf, dass das jeweilige Deutungsmuster als Bindeglied zwischen der angebotenen Struktur und der ausgeführten Handlung verstanden werden kann. Die Deutungsmuster ermöglichen einen konstruktiven Umgang mit der in Schleswig-Holstein vorherrschenden Wahlsituation. „Deutungsmuster organisieren vorgängig die in Krisenlösungen konstituierten Erfahrungsgehalte der Alltagspraxis“ (Oevermann 2001b: 18). Sie sind als „Mittel zur Bewältigung von Handlungsproble- 
men“ (Meuser/Sackmann 1992: 16) anzusehen und wirken komplexitätsreduzierend. Angesichts bestimmter Merkmale (beispielsweise der Signalwörter „Training“ bzw. „Universität") werden wesenseigene Erwartungen antizipiert und das Angebot wird auf Kompatibilität zum eigenen Deutungsmuster geprüft. Es konnte gezeigt werden, dass beide Deutungsmuster über ganz unterschiedliche Wahrnehmungsprioritäten und Bewertungsmaßstäbe verfügen.

Im Anschluss an die Wahl vollzieht sich im Zuge eines Hineingleitens in das institutionelle Angebot eine gegenseitige Bestätigung der Deutungsmuster durch die Teilnehmenden. Dadurch werden die Deutungsmuster erneut verfestigt und stabilisieren ihrerseits wiederum die Strukturmerkmale bzw. die implizite Konstruktion des institutionellen Qualifizierungsangebotes.

Offenkundig wurde durch die rekonstruierten Deutungsmuster, dass das berufliche Handeln im Schulleitungsamt unterschiedlich ausgedeutet werden kann. Es tut sich ein Spannungsfeld zwischen hierarchischer Autorität und personaler Egalität auf, welches im Alltag ausgehandelt werden muss. Um dieses im Rahmen der vorgefundenen Struktur zu bearbeiten, müssen potentielle Schulleitungen reflexionsfähig sein. Auch das Spannungsverhältnis zwischen wissenschaftlichem Expertentum und vorwiegend praxisorientiertem Akteur muss mittels Selbstreflexion erkannt und überdacht werden. In diesem Sinne können die hier dargelegten Deutungsmuster einen Anlass zur Reflexion über den eigenen Standpunkt geben und dazu auffordern, diesen kritisch auch auf Handlungsalternativen für die künftige Praxis zu hinterfragen. Dies ist insbesondere vor dem Hintergrund relevant, dass alle befragten Personen ihre Deutungsmuster - entsprechend der Oevermannschen Sichtweise, Deutungsmuster seien „Routinen, die sich in langer Bewährung eingeschliffen haben und wie implizite Theorien verselbständigt operieren“ (Oevermann 2001a: 38) - nicht auf den Geltungscharakter hin hinterfragt haben.

Inwieweit sich die verinnerlichten Auffassungen von Führung und der Bedeutsamkeit von Wissenschaftlichkeit letztlich in praxi im Schulleitungshandeln dieser Personen niederschlagen, kann erst durch weitere Forschung eruiert werden. Offen bleiben muss zudem, in welcher Ausprägung die jeweilige Qualifizierungsmaßnahme realiter Handeln auf Basis von Wissenschaftlichkeit respektive spezifische handlungspraktische Kompetenzen herauszubilden vermag und ob Differenzen im Schulleitungs- und Führungshandeln von Personen, die durch das „TVaS“ respektive den Masterstudiengang qualifiziert wurden, empirisch fassbar werden.

\section{Autorenangaben}

Dr. phil. Wiebke Bobeth-Neumann

Fachbereich Erziehungswissenschaften,

Universität Hamburg

wiebke.bobeth@uni-hamburg.de 


\section{Literatur}

Allgemeine Schulleitungsverband Deutschlands (ASD) - Verband deutscher Schulleitungen e.V. (Hrsg.) (1999): Schulleitung in Deutschland. Ein Berufsbild in Entwicklung. Stuttgart: Raabe.

Baumert, J./Leschinsky, A. (1986): Berufliches Selbstverständnis und Einflußmöglichkeiten von Schulleitern: Ergebnisse einer Schulleiterbefragung. Berlin: Max-Planck-Institut für Bildungsforschung.

Bobeth-Neumann, W. (2013): Karriere „Grundschulleitung“ Über den Einfluss des Geschlechts beim beruflichen Aufstieg ins Schulleitungsamt. Bielefeld: transcript Verlag.

Bohnsack, R./Nentwig-Gesemann, I./Nohl,A.-M. (Hrsg.) (2001): Die dokumentarische Methode und ihre Forschungspraxis. Grundlagen qualitativer Sozialforschung. Opladen: Leske + Budrich.

Brohm, M. (2004): Management des Wandels. In: Böttcher, W./Terhart, E. (Hrsg.): Organisationstheorie in pädagogischen Feldern. Wiesbaden: VS Verlag für Sozialwissenschaften, S. 173-190.

Giddens, A. (1984a): Interpretative Soziologie. Eine kritische Einführung. Frankfurt/Main: Campus-Verlag.

Giddens, A. (1984b): The constitution of society: Outline of the theory of structuration. Cambridge: Polity Press.

Giddens, A. (1988): Die Konstitution der Gesellschaft. Grundzüge einer Theorie der Strukturierung. Frankfurt/Main: Campus-Verlag.

Giddens, A. (1997): Die Konstitution der Gesellschaft. Grundzüge einer Theorie der Strukturierung. 3. Aufl. Frankfurt/Main: Campus-Verlag.

Huber, S. G./Wissinger, J. (2002) (Hrsg.): Schulleitung - Forschung und Qualifizierung. Opladen: Leske \& Budrich.

Huber, S. G. (2003): Qualifizierung von Schulleiterinnen und Schulleitern im internationalen Vergleich: eine Untersuchung in 15 Ländern zur Professionalisierung von pädagogischen Führungskräften für Schulen. Kronach: Link Verlag.

Meuser, M./Sackmann, R. (1992) (Hrsg.): Analyse sozialer Deutungsmuster. Pfaffenweiler: Centaurus-Verlagsgesellschaft.

Neuberger, O. (1995): Mikropolitik: der alltägliche Aufbau und Einsatz von Macht in Organisationen. Stuttgart: Enke Verlag.

Nohl, A.-M. (2008): Interview und dokumentarische Methode. 2. Aufl. Wiesbaden: VS Verlag.

Oevermann, U. (2001a): Die Struktur sozialer Deutungsmuster - Versuch einer Aktualisierung. In: Sozialer Sinn. Zeitschrift für hermeneutische Sozialforschung 2,1, S. 35-82.

Oevermann, U. (2001b): Zur Analyse der Struktur von sozialen Deutungsmustern. In: Sozialer Sinn. Zeitschrift für hermeneutische Sozialforschung 2,1, S. 3-34.

Rahm, S. (2005): Einführung in die Theorie der Schulentwicklung. Weinheim, Basel: Beltz Verlag.

Schratz, M. (1998): Schulleitung als change agent: Vom Verwalten zum Gestalten von Schule. In: Altrichter, H./Schley, W./Schratz, M. (Hrsg.): Handbuch zur Schulentwicklung. Innsbruck: Studienverlag, S. 160-189.

Ullrich, C. G. (1999): Deutungsmusteranalyse und diskursives Interview. In: Zeitschrift für Soziologie 28,6, S. 429-447.

Walgenbach, P. (2006): Die Strukturationstheorie. In: Kieser, A./Ebers, M. (Hrsg.): Organisationstheorien. Stuttgart: Kohlhammer Verlag, S. 403-426.

Wernet, A. (2003): Pädagogische Permissivität: schulische Sozialisation und pädagogisches Handeln jenseits der Professionalisierungsfrage. Opladen: Leske + Budrich.

Wolfmeyer, P. (1981): Die schulinterne Verwaltungstätigkeit der Lehrer: eine empirische Untersuchung in Grund-, Haupt-, Real- und beruflichen Schulen sowie den Gymnasien NordrheinWestfalens. Kastellaun/Hunsrück: Henn. 